\title{
Average Case Performance of Replicator Dynamics in Potential Games via Computing Regions of Attraction
}

IOANNIS PANAGEAS, Georgia Institute of Technology

GEORGIOS PILIOURAS, Singapore University of Technology and Design

What does it mean to fully understand the behavior of a network of adaptive agents? The golden standard typically is the behavior of learning dynamics in potential games, where many evolutionary dynamics, e.g., replicator dynamics, are known to converge to sets of equilibria. Even in such classic settings many questions remain unanswered. We examine issues such as:

- Point-wise convergence: Does the system always equilibrate, even in the presence of continuums of equilibria?

- Computing regions of attraction: Given point-wise convergence can we compute the region of asymptotic stability of each equilibrium (e.g., estimate its volume, geometry)?

- System invariants: Invariant functions remain constant along every system trajectory. This notion is orthogonal to the game theoretic concept of a potential function, which always strictly increases/decreases along system trajectories. Do dynamics in potential games exhibit invariant functions? If so, how many? How do these functions look like?

Based on these geometric characterizations, we propose a novel quantitative framework for analyzing the efficiency of potential games with many equilibria. The predictions of different equilibria are weighted by their probability to arise under evolutionary dynamics given uniformly random initial conditions. This average case analysis is shown to offer novel insights in classic game theoretic challenges, including quantifying the risk dominance in stag-hunt games and allowing for more nuanced performance analysis in networked coordination and congestion games with large gaps between price of stability and price of anarchy.

CCS Concepts: $\bullet$ Theory of computation $\rightarrow$ Solution concepts in game theory; Convergence and learning in games;

Additional Key Words and Phrases: Dynamical Systems; Congestion Games; Replicator; Price of Anarchy

\section{INTRODUCTION}

The study of game dynamics is a basic staple of game theory with several books dedicated exclusively to it [Cesa-Bianchi and Lugoisi 2006; Fudenberg and Levine 1998; Hofbauer and Sigmund 1998; Sandholm 2010; Young 2004]. Historically, the golden standard for classifying the behavior of learning dynamics in games has been to establish convergence to equilibria. Thus, it is hardly surprising that a significant part of the work on learning in games focuses on potential games (and slight generalizations thereof) where many dynamics (e.g., replicator, smooth fictitious play) are known to converge to equilibrium sets. The structure of the convergence proofs is essentially

Ioannis Panageas was supported by NSF EAGER award grants CCF-1415496 and CCF-1415498. Georgios Piliouras was supported by SUTD grant SRG ESD 2015 097. Part of this work was done while both authors were visiting Simons Institute for the Theory of Computing at Berkeley. The full version of this paper is available at arxiv.org/abs/1403.3885. The theorem numbering here matches the full version. Authors' addresses: I. Panageas, Georgia Institute of Technology; email: ioannis@gatech.edu; G. Piliouras, Singapore University of Technology and Design; email: georgios@sutd.edu.sg.

Permission to make digital or hard copies of all or part of this work for personal or classroom use is granted without fee provided that copies are not made or distributed for profit or commercial advantage and that copies bear this notice and the full citation on the first page. Copyrights for components of this work owned by others than ACM must be honored. Abstracting with credit is permitted. To copy otherwise, or republish, to post on servers or to redistribute to lists, requires prior specific permission and/or a fee. Request permissions from permissions@acm.org.

EC'16, July 24-28, 2016, Maastricht, The Netherlands. Copyright (C) 2016 ACM 978-1-4503-3936-0/16/07 ...\$15.00.

http://dx.doi.org/10.1145/10.1145/2940716.2940784 
universal across different learning dynamics and boils down to identifying a Lyapunov/potential function that strictly decreases along any nontrivial trajectory. In potential games, as their name suggests, this function is part of the description of the game and precisely guides self-interested dynamics towards critical points of these functions that correspond to equilibria of the learning process.

Potential games are also isomorphic to congestion games [Monderer and Shapley 1996]. Congestion games have been instrumental in the study of efficiency issues in games. They are amongst the most extensively studied class of games from the perspective of price of anarchy and price of stability with many tight characterization results for different subclasses of games (e.g., linear congestion games [Roughgarden 2009], symmetric load balancing [Nisan et al. 2007] and references therein).

Given this extensive treatment of such a classic class of games it would seem, at a first glance, that our understanding of these systems is more or less complete. We show that this is far from the case. We focus on simple systems where replicator dynamic, arguably one of the most well studied game dynamics, is applied to linear congestion games and (network) coordination games. We resolve a number of basic open questions in the following results:

A) Point-wise convergence to equilibrium: In the case of linear congestion games and (network) coordination games we prove convergence to equilibrium instead of equilibrium sets. Convergence to equilibrium sets implies that the distance of system trajectories from the sets of equilibria converges to zero. On the other hand, convergence to equilibrium, also referred to as point-wise convergence, implies that every system trajectory has a unique limit point, which is an equilibrium. In games with continuums of equilibria, (e.g., $n$ balls $n$ bins games ${ }^{1}$ with $n \geq 4$ ), the first statement is more inclusive that the second. In fact, system equilibration is not implied by set-wise convergence, and the limit set of a trajectory may have complex topology (e.g., the limit of social welfare may not be well defined). Despite numerous positive convergence results in classes of congestion games ([Ackermann et al. 2009; Berenbrink et al. 2007, 2012; Even-Dar and Mansour 2005; Fotakis et al. 2008]), this is the first to our knowledge result about deterministic point-wise convergence for any concurrent dynamic. This argument is based on combining global Lyapunov functions arguments with local information theoretic Lyapunov functions around each equilibrium.

B) Global stability analysis: Although the point-wise convergence result is interesting in itself, it critically enables all other results in the paper. Specifically, we establish that modulo point-wise convergence, all but a zero measure set of initial conditions converge to equilibrium points which are (linearly) stable (i.e., their Jacobian has no eigenvalue with positive real part). This is a technical result that combines game theoretic arguments with tools from dynamical systems (Center-Stable Manifold theorem) and analysis (Lindelöf's lemma). Kleinberg et al. [2009] has established that all such equilibria must satisfy a refined game theoretic property and known as weakly stability. A Nash equilibrium is weakly stable if given any two randomizing agents, fixing one of the agents to choosing one of his strategies with probability one, leaves the other agent indifferent between the strategies in his support. This condition is easy to work with (does not require computing eigenvalues) and sometimes (along with the global stability result) already suffices to make a unique prediction about the resulting system performance.

C) Invariant functions: Sometimes a game may have multiple (weakly) stable equilibria. In this case we would like to be able to predict which one will arise given a specific (or maybe a randomly chosen) initial condition. Systems invariants allows

\footnotetext{
${ }^{1}$ These are symmetric load balancing games with $n$ agents and $n$ machines where the cost function of each machine is the identity function.
} 
us to do exactly that. A system invariant is a function defined over the system state space such that it remains constant along every system trajectory. Establishing invariant properties of replicator dynamics in generalized zero-sum games has helped prove interesting topological properties of the system trajectories such as (near) cycles [Papadimitriou and Piliouras 2016; Piliouras et al. 2014; Piliouras and Shamma 2014]. In the case of bipartite coordination games with fully mixed Nash equilibria, we can establish similar invariant functions. Specifically, the difference between the sum of the Kullback-Leibler (K-L) divergences of the evolving mixed strategies of the agents on the left partition from their fully mixed Nash equilibrium strategy and the respective term for the agents in the right partition remains constant along any trajectory. In the special case of star graphs, we show how to produce $n$ such invariants where $n$ is the degree of the star. This allows for creating efficient oracles for predicting to which Nash equilibrium the system converges provably for any initial condition without simulating explicitly the system trajectory.

Applications: The tools that we have developed allow for novel insights in classic and well studied class of games. We group our results into two clusters, average case performance analysis and estimating risk dominance/regions of attraction.

Average Case Performance: We propose a novel quantitative framework for analyzing the efficiency of potential games with many equilibria. Informally, we define the expected system performance as the weighted average of the social costs of all equilibria where the weight of each equilibrium is proportional to the volume (or more generally measure) of its region of attraction.

The main idea is as follows: The agents start participating in the game having some prior beliefs about which are the best actions for them. We will typically assume that the initial beliefs are chosen according to a uniform prior given that we want to assume no knowledge about the agents' internal beliefs ${ }^{2}$. Given this initial condition the agents start interacting through the game and update their beliefs (i.e., their randomized strategies) up until they reach equilibrium. At this point the measure of the region of attraction of an equilibrium captures exactly the likelihood that we will converge to that state. So the average case performance computes, as its names suggests, what will be the resulting system performance on average. As is typical in algorithmic game theory, we can normalize this quantity by dividing with the performance of the optimal state. We define this ratio as the average price of anarchy. In our convergent systems it always lies between the price of stability and the price of anarchy. We analyze the average price of anarchy in a number of settings which include, $n$ balls $n$ bins games, symmetric linear load balancing games (with agents of equal weights) ${ }^{3}$, parametric versions of coordination games as well as star network extensions of them. These are games with large gaps between the price of stability and price of anarchy and replicator is shown to be able to zero in on the good equilibria with high enough probability so that the average price of anarchy is always a small constant. This measure of performance could help explain why some games are easy in practice, despite having large price of anarchy. We aggregate these results below:

\begin{tabular}{|l|c|c|c|c|c|}
\hline & Average PoA & Techniques & PoS & Pure PoA & PoA \\
\hline$n$ balls $n$ bins game & $\mathbf{1}$ & A \& B & 1 & 1 & $\Theta(\log n / \log \log n)$ \\
\hline Symmetric Load Balancing & {$[\mathbf{1 , 1 . 5}]$} & A \& B & 1 & 1 & $\Omega(\log n / \log \log n)$ \\
\hline$w$-Coordination Game & {$[1.15, \mathbf{1 . 2 1}]$} & $\mathbf{A ~ \& ~ B ~ \& ~ C ~}$ & 1 & $\Theta(w)$ & $\Theta(w)$ \\
\hline$N$-Star $w$-Coordination Game & {$[\mathbf{1 . 1 5}, \mathbf{1 . 8 6 8}]$} & $\mathbf{A ~ \& ~ B ~ \& ~ C ~}$ & 1 & $\Theta(w)$ & $\Theta(w)$ \\
\hline
\end{tabular}

\footnotetext{
${ }^{2}$ Our techniques extend to arbitrarily correlated beliefs, any prior over initial mixed strategies.

${ }^{3} \mathrm{We}$ focus mostly on the makespan as a measure of social cost.
} 
Risk dominance / Regions of attraction: Risk dominance is an equilibrium refinement process that centers around uncertainty about opponent behavior. A Nash equilibrium is considered risk dominant if it has the largest basin of attraction ${ }^{4}$. The benchmark example is the Stag Hunt game, shown in figure 1(a). In such symmetric $2 \times 2$ coordination games a strategy is risk dominant if it is a best response to the uniformly random strategy of the opponent. We show that the likelihood of the risk dominant equilibrium of the Stag Hunt game is $\frac{1}{27}(9+2 \sqrt{3} \pi) \approx 0.7364$ (instead of merely knowing that it is at least $1 / 2$, see figure 2). The size of the region of attraction of the risk dominated equilibrium is 0.2636 , whereas the mixed equilibrium has region of attraction of zero measure. Moving to networks of coordination games, we show how to construct an oracle that predicts the limit behavior of an arbitrary initial condition, in the case of coordination games played over a star network with $N$ agents. This is the most economic class of games that exhibits two characteristics that intuitively seem to pose intractable obstacles to the quantitative analysis of nonlinear systems: i) they have (arbitrarily many) free variables, ii) they exhibit a continuum of equilibria.

\section{RELATED WORK}

Set-wise convergence in congestion/potential games: A number of positive convergence results have been established for concurrent dynamics [Ackermann et al. 2009; Berenbrink et al. 2007, 2012; Even-Dar and Mansour 2005; Fotakis et al. 2008; Kleinberg et al. 2009], however, they usually depend on strong assumptions about network structure (e.g., load balancing games) and/or symmetry of available strategies and/or are probabilistic in nature and/or establish convergence to approximate equilibria. On the contrary our convergence results are deterministic, hold for any network structure and are point-wise.

Learning as a refinement/prediction mechanism in game theory: Price of anarchy-like bounds in potential games using equilibrium stability refinements (e.g., stochastically stable states) have been explored before [Ackermann et al. 2009; Asadpour and Saberi 2009; Chung et al. 2008]. Our approach and techniques are more expansive in scope, since they also allow for computing the actual likelihoods of each equilibrium as well as the topology of the regions of attractions of different equilibria.

We build upon positive performance results for replicator dynamics (and discretetime variants). The key reference is [Kleinberg et al. 2009], where many key ideas including the fact that replicator dynamics can significantly outperform worst case equilibria were introduced. This stability analysis can be generalized to deterministic variants of replicator [Mehta et al. 2015]. Replicator can outperform even best case equilibria by converging to cycles [Kleinberg et al. 2011; Ligett and Piliouras 2011].

In independent parallel work [Zhang and Hofbauer 2014] examine equilibrium selection issues in $2 \times 2$ coordination games for replicator dynamics, however, their techniques do not scale to larger games. Analyzing the regions of attraction for (variants of) replicator dynamics in (time-evolving) games raises interesting computational questions relevant to mathematical biology [Mehta et al. 2015; Mehta et al. 2014].

Papadimitriou and Piliouras [2016] show how to use elements from the theory of topology of dynamical systems such as chain recurrent sets to analyze learning dynamics in games. This solution concept generalizes the notion of Nash equilibrium and captures the actual limit behavior of game dynamics. Combining the ideas of regions of attraction with chain recurrent sets opens up interesting directions for average case analysis of learning dynamics in non-potential games.

\footnotetext{
${ }^{4}$ Although risk dominance [Harsanyi and Selten 1988] was originally introduced as a hypothetical model of the method by which perfectly rational players select their actions, it may also be interpreted [Myerson $1991]$ as the result of evolutionary processes.
} 
Regions of Attraction \& Gradient Dynamics in Non-Convex Optimization: In recent work, [Lee et al. 2016; Panageas and Piliouras 2016] have shown how to combine tools from dynamical systems theory to understand the behavior of one of the most classic optimization heuristics, deterministic discrete-time fixed step-size gradient dynamics in general non-convex fitness landscapes. Specifically, it is argued that saddle points (non-local minima fixed points) have regions of attraction of zero measure and hence gradient dynamics typically converge to local minima.

\section{Organization of the paper}

The rest of the paper is organized as follows. In Section 3 we provide definitions in regards to dynamical systems, congestion and network coordination games and the average price of anarchy. In Section 4 we establish point-wise convergence of replicator dynamics for congestion and network coordination games and we develop the mathematical machinery necessary for approximating the average price of anarchy. In Section 5 we present our average price of anarchy results. All the missing proofs can be found in the full version of this paper which is available at arxiv.org/abs/1403.3885.

\section{DEFINITIONS AND BASIC TOOLS}

\section{Notation}

We use boldface letters, e.g. $\mathbf{x}$, to denote vectors and denote a vector's $i^{t h}$ coordinate by $x_{i}$. We use $\mathbf{x}_{-i}$ to denote $\mathbf{x}$ after removing coordinate $i$-th. For a function $f$, we denote by $f^{n}$ the composition of $f$ with itself $n$ times, namely $\underbrace{f \circ f \circ \cdots \circ f}_{n \text { times }}$. We use $\mathcal{J}[\mathbf{x}]$ to denote the Jacobian matrix (of some function clear from the context) at the point $\mathbf{x}$.

\subsection{Dynamical Systems}

Let $f: \mathcal{S} \rightarrow \mathbb{R}^{n}$ be continuously differentiable with $\mathcal{S} \subset \mathbb{R}^{n}, \mathcal{S}$ a compact set. We examine continuous (time) dynamical systems of the form

$$
\frac{d \mathbf{x}}{d t}=f(\mathbf{x}) .
$$

Since $\mathcal{S}$ is compact and $f$ continuously differentiable, $f$ is globally Lipschitz in $\mathcal{S}$ and the ordinary differential equation (1) along with the initial condition $\mathrm{x}(0)=\mathrm{x}_{0} \in \mathcal{S}$ has a unique solution for $t \in \mathbb{R}$ and we can present it by $\phi\left(t, \mathbf{x}_{0}\right)$, called the flow of the system. $\phi_{t}\left(\mathbf{x}_{0}\right):=\phi\left(t, \mathbf{x}_{0}\right)$ corresponds to a function of time which captures the trajectory of the system with $\mathrm{x}_{0}$ the given starting point. It is continuously differentiable, its inverse exists (denoted by $\phi_{-t}\left(\mathbf{x}_{0}\right)$ ) and is also continuously differentiable (called diffeomorphism). It is also true that $\phi_{t} \circ \phi_{s}=\phi_{t+s}$ for $t, s \in \mathbb{R}$ and therefore $\phi_{k}=\phi_{1}^{k}$ for $k \in \mathbb{N}$ (composition of $\phi_{1} k$ times). $\mathbf{x}_{0} \in \mathcal{S}$ is called an equilibrium if $f\left(\mathbf{x}_{0}\right)=\mathbf{0}$. In that case holds $\phi_{t}\left(\mathbf{x}_{0}\right)=\mathbf{x}_{0}$ for all $t \in \mathbb{R}$, i.e., $\mathbf{x}_{0}$ is a fixed point of the function $\phi_{t}(\mathbf{x})$ for all $t \in \mathbb{R}$. We call $\mathbf{x}_{0}$ linearly stable if the eigenvalues of the Jacobian $\mathcal{J}\left[\mathbf{x}_{0}\right]$ of $f$ (at the fixed point $\mathrm{x}_{0}$ ) have non-positive real part.

A Lyapunov (or potential) function $V: \mathcal{S} \rightarrow \mathbb{R}$ is a function that strictly decreases along every non-trivial trajectory of the dynamical system. Formally, for continuous time dynamical systems it holds that $\frac{d V}{d t} \leq 0$ with equality only when $f(\mathbf{x})=\mathbf{0}$. For more information on dynamical systems see [Perko 1991].

\subsection{Average Performance of a system}

Let $\mu$ be the Lebesgue measure on $\mathbb{R}^{n}$ and assume that $\mu(\mathcal{S})>0$. Given a dynamical system (continuous time) we assume that $\lim _{t \rightarrow \infty} \phi_{t}(\mathbf{x})$ exists for all $\mathrm{x} \in \mathcal{S}$ (the limit is called a limit point); the system converges point-wise for all initial conditions. In 
this case, continuity implies that every trajectory converges to some equilibrium (fixed point) of the dynamics. ${ }^{5}$

We would like to understand the average (long-term) behavior of the convergent system (e.g., if the initial condition is chosen uniformly at random from $\mathcal{S}$ ). Intuitively, since the system converges to fixed points, we would like each fixed point to be assigned weight proportional to its region of attraction. We define the region of attraction of a fixed point $\mathrm{x}_{0}$ by $R_{\mathbf{x}_{0}}=\left\{\mathrm{x} \in \mathcal{S}: \lim _{t \rightarrow \infty} \phi_{t}(\mathbf{x})=\mathrm{x}_{0}\right\}$, namely the set of starting points so that the dynamic converges to $\mathbf{x}_{0}$. Let $\psi(\mathbf{x})=\lim _{t \rightarrow \infty} \phi_{t}(\mathbf{x})$, i.e., $\psi$ maps each starting point $\mathbf{x}$ to the limit of the $\phi_{t}(\mathbf{x})$. It turns out that $\psi$ is measurable (see Lemma 7.1 in the full version) and we can define the average (long-term) performance of the system under some (utilitly/cost) function $u$. Let $u: \mathcal{S} \rightarrow \mathbb{R}$ be continuous then the average (case) performance of a system is defined as

$$
\operatorname{acp}_{u}:=\frac{\int_{\mathcal{S}} u \circ \psi d \mu}{\mu(\mathcal{S})}=\mathbb{E}_{\mathbf{x} \sim U(\mathcal{S})}[u(\psi(\mathbf{x}))],
$$

where $U(\mathcal{S})$ is the uniform distribution on $\mathcal{S}$. $u$ quantifies the quality of the points $\mathbf{x} \in \mathcal{S}$ (e.g., social welfare in games). Observe that if $m:=\min _{\mathbf{x} \in \mathrm{FP}} u(\mathbf{x}), M:=\max _{\mathbf{x} \in \mathrm{FP}} u(\mathbf{x})$ where FP denotes the set of fixed points ${ }^{6}$ then $m \leq \operatorname{acp}_{u} \leq M$ (a). We believe that computing/approximating the average case performance is an important step towards understanding the actual behavior of a system.

To see the connection with game theory, think of $\mathcal{S}$ as the set of mixed (randomized) strategies, a fixed point with region of attraction of positive measure as a Nash equilibrium and $u$ as the social cost/welfare. In this case, integral (2) becomes a weighted average among the social cost/welfare of the Nash equilibria. The average case performance is sandwiched between the values (of the social cost/welfare) at the worst, best Nash equilibrium.

We use (continuous time) replicator dynamics on congestion and network coordination games as our benchmark. In this case, the set of Nash equilibria is a subset of the set of fixed points. Nevertheless, we can show that the dynamics converge point-wise and finally that Nash equilibria are the only fixed points whose region of attraction may be of positive Lebesgue measure. Later in this section we define the notion of $a v$ erage price of anarchy which is essentially a scaled version of average performance, defined particularly for games.

Remark 3.1 (Generalizations of average case performance). The definition of average case performance can be used for any point-wise convergent discrete time dynamical systems (function $\psi(\mathbf{x})$ will be equal to $\lim _{k \rightarrow \infty} g^{k}(\mathbf{x})$ where $g$ is the rule of the discrete dynamics). Also, different measures of efficiency can be defined where the initial condition follows some distribution other than the uniform. Generally, the distribution over initial conditions, the notion of (social) utility/cost, and the dynamic can all be treated as parameters of this performance measure.

\subsection{Replicator Dynamics on Congestion/Network Coordination Games}

Congestion Games. A congestion game is defined by the tuple $\left(\mathcal{N} ; \mathcal{E} ;\left(S_{i}\right)_{i \in \mathcal{N}} ;\left(c_{e}\right)_{e \in \mathcal{E}}\right)$ where $\mathcal{N}$ is the set of agents (with $N=|\mathcal{N}|$ ), $\mathcal{E}$ is a set of resources (also known as edges or bins or facilities), and each player $i$ has a set $S_{i}$ of subsets of $\mathcal{E}\left(S_{i} \subseteq 2^{\mathcal{E}}\right)$ and $\left|S_{i}\right| \geq 2$. Each strategy $s_{i} \in S_{i}$ is a set of edges (a path), and $c_{e}$ is a cost (negative utility) function associated with facility $e$. We will also use small greek characters like $\gamma, \delta$ to denote different strategies/paths. For a strategy profile $\mathbf{s}=\left(s_{1}, s_{2}, \ldots, s_{N}\right)$, the

\footnotetext{
${ }^{5}$ If $\lim _{t \rightarrow \infty} h^{t}(\mathbf{x})=\mathbf{y}$ and $h$ continuous then $h(\mathbf{y})=\mathbf{y}$. Set $h:=\phi_{1}$.

${ }^{6}$ The set of fixed points in $\mathcal{S}$ is closed.
} 
cost of player $i$ is given by $c_{i}(\mathbf{s})=\sum_{e \in s_{i}} c_{e}\left(\ell_{e}(\mathbf{s})\right)$, where $\ell_{e}(\mathbf{s})$ is the number of players using $e$ in $\mathbf{s}$ (the load of edge $e$ ). In linear congestion games, the latency functions are of the form $c_{e}(x)=a_{e} x+b_{e}$ where $a_{e}, b_{e} \geq 0$. Measures of social cost (sc(s)) include the makespan, which is equal to the cost of the most expensive path and the sum of the costs of all the agents.

Network (Polymatrix) Coordination Games. A coordination (or partnership) game is a two player game where in each strategy outcome both agents receive the same utility. In other words, if we flip the sign of the utility of the first agent then we get a zero-sum game. An $N$-player polymatrix (network) coordination game is defined by an undirected graph $G(V, E)$ with $|V|=N$ vertices and each vertex corresponds to a player. An edge $(i, j) \in E(G)$ corresponds to a coordination game between players $i, j$. We assume that we have the same strategy space $S$ for every edge. Let $A_{i j}$ be the payoff matrix for the game between players $i, j$ and $A_{i j}^{\gamma \delta}$ be the payoff for both (coordination) if $i, j$ choose strategies $\gamma, \delta$ respectively. The set of players will be denoted by $N$ and the set of neighbors of player $i$ will be denoted by $N(i)$. For a strategy profile $\mathbf{s}=\left(s_{1}, s_{2}, \ldots, s_{N}\right)$, the utility of player $i$ is given by $u_{i}(\mathbf{s})=\sum_{j \in N(i)} A_{i j}^{s_{i} s_{j}}$. The social welfare of a state $\mathbf{s}$ corresponds to the sum of the utilities of all the agents $s w(\mathbf{s})=\sum_{i \in V} u_{i}(\mathbf{s})$.

The price of anarchy is defined as: $\mathrm{PoA}=\frac{\max _{\mathbf{s} \in \mathrm{NE}} \operatorname{Social} \operatorname{Cost}(\mathbf{s})}{\min _{\mathbf{s}^{*} \in \times_{i} S_{i}} \operatorname{Social} \operatorname{Cost}\left(\mathbf{s}^{*}\right)}$ for cost functions and similarly $\mathrm{PoA}=\frac{\max _{\mathbf{s}^{*} \in \times_{i} S_{i}} \text { Social Welfare }\left(\mathbf{s}^{*}\right)}{\min _{\mathbf{s} \in \mathrm{NE}} \text { Social Welfare }(\mathbf{s})}$ for utilities. ${ }^{7}$

We denote by $\Delta\left(S_{i}\right)=\left\{\mathbf{p} \geq \mathbf{0}: \sum_{\gamma} p_{i \gamma}=1\right\}$ the set of mixed (randomized) strategies of player $i$ and $\Delta=\times_{i} \Delta\left(S_{i}\right)$ the set of mixed strategies of all players. For congestion games we use $c_{i \gamma}=\mathbb{E}_{s_{-i} \sim \mathbf{p}_{-i}} c_{i}\left(\gamma, \mathbf{s}_{-i}\right)$ to denote the expected cost of player $i$ given that he chooses strategy $\gamma$ and $\hat{c}_{i}=\sum_{\delta \in S_{i}} p_{i \delta} c_{i \delta}$ to denote his expected cost. Similarly, for network coordination games we use $u_{i \gamma}=\mathbb{E}_{s_{-i} \sim \mathbf{p}_{-i}} u_{i}\left(\gamma, \mathbf{s}_{-i}\right)$ to denote the expected utility of player $i$ given that he chooses strategy $\gamma$ and $\hat{u}_{i}=\sum_{\delta \in S_{i}} p_{i \delta} u_{i \delta}$ to denote his expected utility.

Replicator Dynamics. Replicator dynamics is described by the following system of differential equations adjusted to cost games (e.g., congestion games) and utility games (e.g., network coordination games) respectively:

$$
\frac{d p_{i \gamma}}{d t}=p_{i \gamma}\left(\hat{c}_{i}-c_{i \gamma}\right), \quad \frac{d p_{i \gamma}}{d t}=p_{i \gamma}\left(u_{i \gamma}-\hat{u}_{i}\right)
$$

for each $i \in \mathcal{N}, \gamma \in S_{i}$. Observe that if $\hat{c}_{i}>c_{i \gamma}$ then $\frac{d p_{i \gamma}}{d t}>0$, i.e., $p_{i \gamma}$ is increasing with time, thus player $i$ tends to increase the probability he chooses strategy $\gamma$. Similarly if $\hat{c}_{i}<c_{i \gamma}$ then $\frac{d p_{i \gamma}}{d t}<0$, i.e., $p_{i \gamma}$ is decreasing with time, thus player $i$ tends to decrease the probability he chooses strategy $\gamma_{.}^{8}$ Replicator dynamics capture similarly rational behavior in the case of network coordination games.

Remark 3.2. The fixed points of replicator dynamics are exactly the set of randomized strategies such that each agent experiences equal costs across all strategies he chooses with positive probability. This is a generalization of the notion of Nash equilibrium, since Nash equilibria furthermore require that any strategy that is played with zero probability must have expected cost at least as high as those strategies which are played with positive probability.

${ }^{7} \mathrm{NE}$ denotes the set of Nash equilibria.

${ }^{8}$ Replicator dynamics describes rational behavior in a sense. 
3.3.1. Definition of average price of anarchy $(A P O A)$. In this section we define the notion of average price of anarchy, following the machinery from Section 3.2. It is natural to set $\mathcal{S}$ to be the product of simplexes $\Delta$, but this does not suffice since $\Delta$ has measure zero in $\mathbb{R}^{M}$, where $M:=\sum_{i}\left|S_{i}\right|$. The reason is that the probabilities sum up to one for each player. To circumvent this issue (since from Section 3.2 we need $\mu(\mathcal{S})>0$ ), we consider a natural projection $g$ of the points $\mathbf{p} \in \Delta$ to $\mathbb{R}^{M-N}$ by excluding a specific but arbitrarily chosen ${ }^{9}$ variable for each player. We denote $g(\Delta)$ the "projected" product of simplexes and the projection of any point $\mathbf{p} \in \Delta$ by $g(\mathbf{p})$ (for example $\left(p_{1, a}, p_{1, b}, p_{1, c}, p_{2, a^{\prime}}, p_{2, b^{\prime}}\right) \rightarrow_{g}\left(p_{1, a}, p_{1, b}, p_{2, a^{\prime}}\right)$ where $p_{1, a}+p_{1, b}+p_{1, c}=1$ and $\left.p_{2, a^{\prime}}+p_{2, b^{\prime}}=1\right)$ ). Given a dynamical system ${ }^{10}$ which is defined in $g(\Delta)$ (projected set of mixed strategies) and which converges point-wise to fixed points, we can define $a c p_{s c}, a c p_{s w}$ to be the average case performance as in Section 3.2. For cost/utility functions the average price of anarchy is defined as follows:

$$
\mathrm{APoA}=\frac{\operatorname{acp}_{\mathrm{sc}}}{\min _{\mathbf{s}^{*} \in \times_{i} S_{i}} \mathbf{s c}\left(\mathbf{s}^{*}\right)}, \quad \mathrm{APoA}=\frac{\max _{\mathbf{s}^{*} \in \times_{i} S_{i}} \operatorname{sw}\left(\mathbf{s}^{*}\right)}{\operatorname{acp}_{\mathrm{sw}}} .
$$

Remark 3.3. The definition of APoA does not rely on the fact that the games are congestion or network coordination nor does it rely on replicator dynamics. Its only requirements is that given a game we apply a dynamic that converges point-wise for all initial mixed strategies. Essentially APoA is a scaled version of the average performance. In the next section we show that replicator dynamics converges point-wise for congestion and network coordination games and also that the fixed points (of replicator on these classes of games) with region of attraction of positive measure are Nash equilibria. In particular APoA is well-defined.

\section{ANALYSIS OF REPLICATOR DYNAMICS IN POTENTIAL GAMES}

In this section we develop the mathematical machinery necessary for computing the average case performance of replicator dynamics in different classes of potential games. Specifically, we establish point-wise convergence of replicator dynamics for linear congestion games and arbitrary networks of coordination games (Theorem 4.1). This allows us to define properly the average case performance which is essentially equal to the weighted sum of the social cost/welfare of all equilibria weighted by the cumulative measure/volume of all initial conditions that converge to each (point-wise). Next, we show that the union of regions of attraction of (locally) unstable equilibria is of measure zero (Theorem 4.5). Combining this result with a game theoretic characterization of (un)stable equilibria in [Kleinberg et al. 2009], known as weakly stable equilibria, establishes that only weakly stable equilibria affect the average case system performance. The analysis here is a strengthening of the techniques of [Kleinberg et al. 2009] to carefully account for the possibility of continuums of unstable equilibria. Finally, we still need to compute for each weakly stable equilibrium the size of its region of attraction. The tool that is necessary for this is to establish invariants for replicator dynamics in different classes of games. We present an information theoretic invariant function (Theorem 4.8) for replicator dynamics for bipartite network coordination games. Such invariant functions have been identified [Piliouras et al. 2014; Piliouras and Shamma 2014] for network extensions of zero sum games [Cai and Daskalakis 2011; Daskalakis and Papadimitriou 2009].

\footnotetext{
${ }^{9}$ Choose an arbitrary ordering of the strategies of each agent and then exclude the last strategy.

${ }^{10} \mathrm{We}$ assume that this system describes the evolution of the mixed strategies of rational agents in some game.
} 


\subsection{Poinwise Convergence}

We show that replicator dynamics converges point-wise for the class of linear congestion and network coordination games. The proof of the theorem has two steps. The first step is standard, utilizes the potential function of the game and establishes convergence to equilibria sets. The critical, second step is to construct a local Lyapunov function in some small neighborhood of a limit point.

THEOREM 4.1. Given any initial condition replicator dynamics converges to a fixed point (point-wise convergence) in all linear congestion and network coordination games.

PROOF. We will prove here the result in the case of linear congestion games. The argument for network coordination games follows similar lines and is in the full version.

We denote by $\hat{c}_{i}$ the expected cost of agent $i$ under mixed strategy profile p. Moreover, $c_{i \gamma}$ is his expected cost when he deviates to strategy $\gamma$ and all other agents still play according to $\mathbf{p}$. We observe that $\Psi(\mathbf{p})=\sum_{i} \hat{c}_{i}+\sum_{i, \gamma} \sum_{e \in \gamma}\left(b_{e}+a_{e}\right) p_{i \gamma}$ is a Lyapunov function since

$$
\frac{\partial \Psi}{\partial p_{i \gamma}}=c_{i \gamma}+\sum_{j \neq i} p_{j \gamma^{\prime}} \frac{\partial c_{j \gamma^{\prime}}}{\partial p_{i \gamma}}+\sum_{e \in \gamma}\left(b_{e}+a_{e}\right)=c_{i \gamma}+\underbrace{\sum_{j \neq i, \gamma^{\prime}} \sum_{e \in \gamma \cap \gamma^{\prime}} a_{e} p_{j \gamma^{\prime}}+\sum_{e \in \gamma}\left(b_{e}+a_{e}\right)}_{c_{i \gamma}}=2 c_{i \gamma}
$$

and hence $\frac{d \Psi}{d t}=\sum_{i, \gamma} \frac{\partial \Psi}{\partial p_{i \gamma}} \frac{d p_{i \gamma}}{d t}=-\sum_{i, \gamma, \gamma^{\prime}} p_{i \gamma} p_{i \gamma^{\prime}}\left(c_{i \gamma}-c_{i \gamma^{\prime}}\right)^{2} \leq 0$, with equality at fixed points. Hence (as in [Kleinberg et al. 2009]) we have convergence to equilibria sets (compact connected sets consisting of fixed points). We will furthermore argue that each trajectory has a unique (equilibrium) limit point.

Let $\mathbf{q}$ be a limit point of the trajectory $\mathbf{p}(t)$. Wlog we can assume that $\mathbf{p}(0)$ is in the interior of $\Delta$ and hence $\mathbf{p}(t)$ is in the interior of $\Delta$ for all $t \in \mathbb{R}$ (we can assume that we start in the interior of $\Delta$ otherwise we can just consider the subgame defined by the strategies that agents play with positive probability.). We have that $\Psi(\mathbf{q}) \leq \Psi(\mathbf{p}(t))$ where the equality holds only if we start at equilibrium. We define the relative entropy $I(\mathbf{p})=-\sum_{i} \sum_{\gamma: q_{i \gamma}>0} q_{i \gamma} \ln \left(p_{i \gamma} / q_{i \gamma}\right) \geq 0$ (Jensen's inequality) and $I(\mathbf{p})=0$ iff $\mathbf{p}=\mathbf{q}$. We denote by $\hat{d}_{i}, d_{i \gamma}$ the expected costs of agent $i$ under the mixed strategies q.

$$
\begin{aligned}
\frac{d I}{d t} & =-\sum_{i} \sum_{\gamma: q_{i \gamma}>0} q_{i \gamma}\left(\hat{c}_{i}-c_{i \gamma}\right)=-\sum_{i} \hat{c}_{i}+\sum_{i, \gamma} q_{i \gamma} c_{i \gamma} \\
& =-\sum_{i} \hat{c}_{i}+\sum_{i, \gamma} \sum_{e \in \gamma}\left(b_{e}+a_{e}\right) q_{i \gamma}+\sum_{i, \gamma} \sum_{j \neq i} \sum_{\gamma^{\prime}} \sum_{e \in \gamma \cap \gamma^{\prime}} a_{e} q_{i \gamma} p_{j \gamma^{\prime}} \\
& =-\sum_{i} \hat{c}_{i}+\sum_{i, \gamma} \sum_{e \in \gamma}\left(b_{e}+a_{e}\right) q_{i \gamma}+\sum_{i, \gamma} \sum_{j \neq i} \sum_{\gamma^{\prime}} \sum_{e \in \gamma \cap \gamma^{\prime}} a_{e} q_{j \gamma^{\prime}} p_{i \gamma} \\
& =-\sum_{i} \hat{c}_{i}+\sum_{i, \gamma} \sum_{e \in \gamma}\left(b_{e}+a_{e}\right) q_{i \gamma}-\sum_{i, \gamma} \sum_{e \in \gamma}\left(b_{e}+a_{e}\right) p_{i \gamma}+\sum_{i, \gamma} p_{i \gamma}\left(d_{i \gamma}\right) \\
& =\sum_{i} \hat{d}_{i}-\sum_{i} \hat{c}_{i}+\sum_{i, \gamma} \sum_{e \in \gamma}\left(b_{e}+a_{e}\right) q_{i \gamma}-\sum_{i, \gamma} \sum_{e \in \gamma}\left(b_{e}+a_{e}\right) p_{i \gamma}-\sum_{i, \gamma} p_{i \gamma}\left(\hat{d}_{i}-d_{i \gamma}\right) \\
& =\Psi(\mathbf{q})-\Psi(\mathbf{p})-\sum_{i, \gamma} p_{i \gamma}\left(\hat{d}_{i}-d_{i \gamma}\right)
\end{aligned}
$$

The rest of the proof follows in a similar way to [Losert and Akin 1983]. 
We break the term $\sum_{i, \gamma} p_{i \gamma}\left(\hat{d}_{i}-d_{i \gamma}\right)$ to positive and negative terms (the zero terms can be ignored), i.e., $\sum_{i, \gamma} p_{i \gamma}\left(\hat{d}_{i}-d_{i \gamma}\right)=\sum_{i, \gamma: \hat{d}_{i}>d_{i \gamma}} p_{i \gamma}\left(\hat{d}_{i}-d_{i \gamma}\right)+\sum_{i, \gamma: \hat{d}_{i}<d_{i \gamma}} p_{i \gamma}\left(\hat{d}_{i}-d_{i \gamma}\right)$

CLAIM 4.2. There exists an $\varepsilon>0$ so that the function $Z(\mathbf{p})=I(\mathbf{p})+2 \sum_{i, \gamma: \hat{d}_{i}<d_{i \gamma}} p_{i, \gamma}$ has $\frac{d Z}{d t}<0$ for $\|\mathbf{p}-\mathbf{q}\|_{1}<\varepsilon$ and $\Psi(\mathbf{q})<\Psi(\mathbf{p})$.

To prove this claim, first assume that $\mathbf{p} \rightarrow \mathbf{q}$. We get $\hat{c}_{i}-c_{i \gamma} \rightarrow \hat{d}_{i}-d_{i \gamma}$ for all $i, \gamma$. Hence for small enough $\varepsilon>0$ with $\|\mathbf{p}-\mathbf{q}\|_{1}<\varepsilon$, we have that $\hat{c}_{i}-c_{i \gamma} \leq \frac{3}{4}\left(\hat{d}_{i}-d_{i \gamma}\right)$ for the terms which $\hat{d}_{i}-d_{i \gamma}<0$. Therefore

$$
\begin{aligned}
\frac{d Z}{d t} & =\Psi(\mathbf{q})-\Psi(\mathbf{p})-\sum_{i, \gamma: \hat{d}_{i}>d_{i \gamma}} p_{i \gamma}\left(\hat{d}_{i}-d_{i \gamma}\right)-\sum_{i, \gamma: \hat{d}_{i}<d_{i \gamma}} p_{i \gamma}\left(\hat{d}_{i}-d_{i \gamma}\right)+2 \sum_{i, \gamma: \hat{d}_{i}<d_{i \gamma}} p_{i \gamma}\left(\hat{c}_{i}-c_{i \gamma}\right) \\
& \leq \Psi(\mathbf{q})-\Psi(\mathbf{p})-\sum_{i, \gamma: \hat{d}_{i}>d_{i \gamma}} p_{i \gamma}\left(\hat{d}_{i}-d_{i \gamma}\right)-\sum_{i, \gamma: \hat{d}_{i}<d_{i \gamma}} p_{i \gamma}\left(\hat{d}_{i}-d_{i \gamma}\right)+3 / 2 \sum_{i, \gamma: \hat{d}_{i}<d_{i \gamma}} p_{i \gamma}\left(\hat{d}_{i}-d_{i \gamma}\right) \\
& =\underbrace{\Psi(\mathbf{q})-\Psi(\mathbf{p})}_{<0}+\underbrace{\sum_{i, \gamma: \hat{d}_{i}>d_{i \gamma}}-p_{i \gamma}\left(\hat{d}_{i}-d_{i \gamma}\right)}_{\leq 0}+1 / 2 \underbrace{\sum_{i, \gamma: \hat{d}_{i}<d_{i \gamma}} p_{i \gamma}\left(\hat{d}_{i}-d_{i \gamma}\right)<0}_{\leq 0}<
\end{aligned}
$$

where we substitute $\frac{p_{i \gamma}}{d t}=p_{i \gamma}\left(\hat{c}_{i}-c_{i \gamma}\right)$ (replicator equations), and the claim is proved. Notice that $Z(\mathbf{p}) \geq 0$ (sum of positive terms and $I(\mathbf{p}) \geq 0$ ) and is zero iff $\mathbf{p}=\mathbf{q}$. (i)

To finish the proof of the theorem, let $\mathbf{q}$ be a non-trivial limit point of $\mathbf{p}(t)$ (i.e., $\mathbf{p}(0)$ is not a fixed point). There exists an increasing sequence of times $t_{i}$, with $t_{n} \rightarrow \infty$ and $\mathbf{p}\left(t_{n}\right) \rightarrow \mathbf{q}$. We consider $\varepsilon^{\prime}$ such that the set $C=\left\{\mathbf{p}: Z(\mathbf{p})<\varepsilon^{\prime}\right\}$ is inside $B=\|\mathbf{p}-\mathbf{q}\|_{1}<$ $\varepsilon$ where $\varepsilon$ is from claim above. Since $\mathbf{p}\left(t_{n}\right) \rightarrow \mathbf{q}$, consider a time $t_{N}$ where $\mathbf{p}\left(t_{N}\right)$ is inside $C$. From the claim above we get that $Z(\mathbf{p})$ is decreasing inside $B$ (and hence inside $C$ ), thus $Z(\mathbf{p}(t)) \leq Z\left(\mathbf{p}\left(t_{N}\right)\right)<\varepsilon^{\prime}$ for all $t \geq t_{N}$, hence the orbit will remain in $C$. By the fact that $Z(\mathbf{p}(t))$ is decreasing in $C$ (claim above) and also $Z\left(\mathbf{p}\left(t_{n}\right)\right) \rightarrow Z(\mathbf{q})=0$ it follows that $Z(\mathbf{p}(t)) \rightarrow 0$ as $t \rightarrow \infty$. Hence $\mathbf{p}(t) \rightarrow \mathbf{q}$ as $t \rightarrow \infty$ using (i).

Remark 4.3. If the fixed points of the dynamics are isolated then a (global) Lyapunov function suffices to show that the system converges point-wise (first step of the proof above). A fixed point $\mathbf{x}_{0}$ is called isolated, if there exists an neighborhood of $\mathbf{x}_{0}$ so that $\mathbf{x}_{0}$ is the unique fixed point in that neighborhood. However, this is not the case even in linear congestion games (see Lemma 8.17 in the full version for examples of linear congestion games with continuums of (Nash) equilibria).

\subsection{Global Stability Analysis}

Replicator dynamics in linear congestion games and network coordination games (as well as any dynamic that converges point-wise) induces a probability distribution over the fixed points. The probability assigned to each fixed point is proportional to the volume of its region of attraction. The fixed points can be exponentially many or even accountable many, but as it is stated below (corollary 4.6), only the weakly stable Nash equilibria have non-zero volumes of attraction.

Definition 4.4. [Kleinberg et al. 2009] A Nash equilibrium is called weakly stable if given any two randomizing agents, fixing one of the agents to choosing one of his strategies with probability one, leaves the other agent indifferent between the strategies in his support. That is a Nash equilibrium $\mathbf{p}$ is weakly stable if for any agents $i, j$ and strategies $\gamma, \gamma^{\prime} \in S_{i}, \delta \in S_{j}$ with $p_{i \gamma}, p_{i \gamma^{\prime}}, p_{j \delta}>0: c_{i}\left(\gamma, \delta, \mathbf{p}_{-i j}\right)=c_{i}\left(\gamma^{\prime}, \delta, \mathbf{p}_{-i j}\right)$. 
[Kleinberg et al. 2009] showed that in congestion games every stable fixed point is a weakly stable Nash equilibrium. The following theorem (that assumes point-wise convergence) has as a corollary that for all but a measure zero set of initial conditions replicator dynamics converges to a weakly stable Nash equilibrium.

THEOREM 4.5. The set of initial conditions for which the replicator converges to unstable fixed points has measure zero in $\Delta$ for linear congestion games and network coordination games.

Sketch. The proof of this theorem relies on dedicated machinery from topology and dynamical systems theory. These tools and the complete proof are presented in detail in the full version of the paper. The main conceptual steps are as follows: First, since the space of mixed strategy profiles (i.e., products of simplices) are of zero measure in their native space we work with projections on subspaces where the set of initial conditions has full measure. Due to a classic theorem in dynamical systems (CenterStable Manifold theorem) we have that the set of initial conditions that stay trapped in a small enough neighborhood of an unstable equilibrium is a zero measure set. Any initial condition that converges (pointwise) to this unstable fixed point must (at some time $t$ ) reach points in this set. All of these initial conditions can thus be covered by a countable union of preimages of the zero measure neighborhood implied by the Center-Stable Manifold theorem. Due to the smoothness of the flow (a technical condition known as diffeomorphism) these preimages must also be of zero measure and the countable union of zero measure sets imply a zero measure region of attraction for each unstable equilibrium. The only remaining hurdle is the case where the game has continuum of equilibria. In this case, although the region of attraction of each equilibrium is of zero measure, their union could have positive measure. Due to compactness of state space, we argue that it suffices to cover each (unstable) equilibrium set with a finite cover of (zero-measure) neighborhoods. At this point standard union bound arguments suffice to complete the argument.

This theorem extends to all congestion games for which the replicator dynamics converges point-wise (e.g., games with finite equilibria). Combining theorem 4.5 with the weakly stable characterization of [Kleinberg et al. 2009] which holds for all congestion/potential games, we get the following:

COROLLARY 4.6. In linear congestion games and network coordination games, for all but a measure zero set of initial conditions, replicator dynamics converges (pointwise) to weakly stable Nash equilibria.

\subsection{Invariant Functions from Information Theory}

We have established that all attracting (i.e., asymptotically stable) fixed points are weakly stable Nash equilibria. We still need to characterize and compute the regions of attraction of these equilibria. The key idea here is to characterize the boundaries of the regions of attraction. This is due to the following theorem:

THEOREM 4.7. [Khalil 1996] If $\mathrm{q}$ is an asymptotically stable equilibrium point for a system $\dot{x}=f(x)$ where $f \in C^{1}$, then its region of attraction $R_{\mathbf{q}}$ is an invariant set whose boundaries are formed by trajectories.

If we identify a (continuous) invariant function $f$, i.e., a function that remains constant on any trajectory, and $\mathbf{q}$ is a (limit) point of the trajectory then the whole trajectory lies on the set $\{\mathbf{x}: f(\mathbf{x})=f(\mathbf{q})\}$. If we identify more invariant functions $f_{1}, f_{2}, \ldots, f_{k}$ then the whole trajectory lies on the set $\left\{\mathbf{x}: f_{1}(\mathbf{x})=f_{1}(\mathbf{q}) \wedge f_{2}(\mathbf{x})=\right.$ $\left.f_{2}(\mathbf{q}) \wedge \cdots \wedge f_{k}(\mathbf{x})=f_{k}(\mathbf{q})\right\}$. By identifying enough invariant functions, we can derive an exact algebraic description of the trajectory. 
By our point-wise convergence result each trajectory converges to an equilibrium. So each point of the state space that does not belong in the region of attraction of a weakly stable equilibrium, must converge to an unstable equilibrium. By computing the (union of) regions of attraction of all unstable equilibria we can understand how they partition the state space into regions of attractions for the asymptotically stable equilibria ${ }^{11}$. All points on the stable manifold of unstable fixed point $q$ lie on the set $\left\{\mathbf{x}: f_{1}(\mathbf{x})=f_{1}(\mathbf{q}) \wedge f_{2}(\mathbf{x})=f_{2}(\mathbf{q}) \wedge \cdots \wedge f_{k}(\mathbf{x})=f_{k}(\mathbf{q})\right\}$ where $f_{1}, \ldots, f_{k}$ the invariant functions of the dynamic. Such descriptions can allow for exact computation of volumes of regions of attraction (Section 5.1), approximate volume computation (Section 5.2), designing efficient oracles for testing if an initial condition belongs to the region of attraction of an equilibrium (Section 5.3), and computing average system performance, amongst other applications.

The following lemma that identifies invariants functions in bipartite coordination games follows straightforwardly from prior work on identifying invariant functions for network generalizations of (linear transformations of) zero-sum games [Piliouras et al. 2014; Piliouras and Shamma 2014]). To prove any such statement it suffices to compute the time derivatives of these functions along any trajectory and show them to be equal to zero.

LEMMA 4.8. Let $\mathbf{p}(t)=\left(\mathbf{p}_{1}(t), \ldots, \mathbf{p}_{N}(t)\right)$ be a trajectory of replicator dynamics when applied to a bipartite network of coordination games that has a fully mixed Nash equilibrium $\mathbf{q}=\left(\mathbf{q}_{1}, \ldots, \mathbf{q}_{N}\right)$ then the function $\sum_{i \in V_{\text {left }}} H\left(\mathbf{q}_{i}, \mathbf{p}_{i}(t)\right)-\sum_{i \in V_{\text {right }}} H\left(\mathbf{q}_{i}, \mathbf{p}_{i}(t)\right)$ is (time-)invariant, where $H(\mathbf{x}, \mathbf{y})=-\sum_{i} x_{i} \ln y_{i}$.

The cross entropy between the Nash q and the state of the system, however is equal to the summation of the K-L divergence between these two distributions and the entropy of q. Since the entropy of q is constant, we derive the following corollary (rephrasing the previous lemma):

COROLlaRY 4.9. Let $\mathbf{p}(t)$ be a trajectory of the replicator dynamic when applied to a bipartite network of coordination games that has a fully mixed Nash equilibrium $\mathbf{q}$ then the $K$-L divergence between $\mathbf{q}$ and the $\mathbf{p}(t)$ is constant, i.e., does not depend on $t$.

\section{APPLICATIONS OF AVERAGE CASE ANALYSIS}

We will use the tools we have developed in the previous section to compute the regions of attractions and find the average case performance of replicator dynamics in classic game theoretic settings. The games we examine are: the Stag Hunt game, (parametric) coordination games, polymatrix coordination games played over a star as well as symmetric linear load balancing games.

\subsection{Exact Quantitative Analysis of Risk Dominance in the Stag Hunt Game}

The Stag Hunt game (figures 1(a)) has two pure Nash, (Stag, Stag) and (Hare, Hare) and a symmetric mixed Nash equilibrium where each agent chooses strategy Hare with probability $2 / 3$. Stag Hunt replicator trajectories are equivalent those of a coordination game with $w=2 .{ }^{12}$ Coordination games are potential games where the potential function in each state is equal to the utility of each agent. Since the mixed Nash is not weakly stable replicator dynamics converges to pure Nash equilibria for all

\footnotetext{
${ }^{11}$ The region of attraction of an unstable equilibrium is referred to as the stable manifold of the (unstable) fixed point.

${ }^{12}$ If both agents reduce their payoff of their first strategy by 4, the replicator trajectories remain invariant. This results to a $w$-coordination game with $w=2$.
} 


\begin{tabular}{c|c|c|}
\multicolumn{1}{c}{} & \multicolumn{1}{c}{ Stag } & \multicolumn{1}{c}{ Hare } \\
\cline { 2 - 3 } Stag & 5,5 & 0,4 \\
\cline { 2 - 3 } Hare & 4,0 & 2,2 \\
\cline { 2 - 3 } & &
\end{tabular}

(a) Stag hunt game.

\begin{tabular}{c|c|c|}
\multicolumn{1}{c}{} & \multicolumn{1}{c}{ Stag } & \multicolumn{1}{c}{ Hare } \\
\cline { 2 - 3 } Stag & 1,1 & 0,0 \\
\cline { 2 - 3 } Hare & 0,0 & w, w \\
\cline { 2 - 3 } & &
\end{tabular}

(b) w-coordination game

Fig. 1.

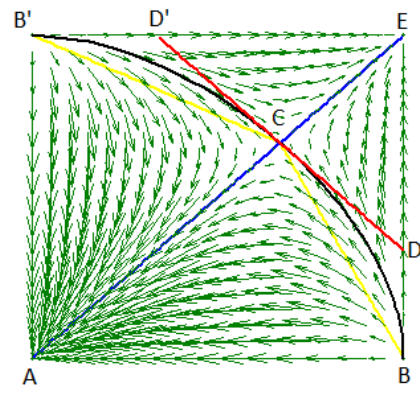

Fig. 2. Vector field of replicator dynamics in Stag Hunt

but a zero measure of initial conditions (Theorem 4.5). When we study the replicator dynamic here, it suffices to examine its projection in the subspace $p_{1 s} \times p_{2 s} \subset(0,1)^{2}$ which captures the evolution of the probability that each agent assigns to strategy Stag (see figure 2). Using the invariant property of lemma 4.8, we compute the size of each region of attraction in this space and thus provide a quantitative analysis of risk dominance in the classic Stag Hunt game.

THEOREM 5.1. The region of attraction of (Hare, Hare) is the subset of $(0,1)^{2}$ that satisfies $p_{2 s}<\frac{1}{2}\left(1-p_{1 s}+\sqrt{1+2 p_{1 s}-3 p_{1 s}^{2}}\right)$ and has Lebesgue measure $\frac{1}{27}(9+2 \sqrt{3} \pi) \approx$ 0.7364. The region of attraction of (Stag, Stag) is the subset of $(0,1)^{2}$ that satisfies $p_{2 s}>$ $\frac{1}{2}\left(1-p_{1 s}+\sqrt{1+2 p_{1 s}-3 p_{1 s}^{2}}\right)$ and has Lebesgue measure $\frac{1}{27}(18-2 \sqrt{3} \pi) \approx 0.2636$. The stable manifold of the mixed Nash equilibrium satisfies the equation $p_{2 s}=\frac{1}{2}\left(1-p_{1 s}+\right.$ $\left.\sqrt{1+2 p_{1 s}-3 p_{1 s}^{2}}\right)$ and has zero Lebesgue measure.

PROOF. In the case of stag hunt games, one can verify in a straightforward manner (via substitution) that $\frac{d\left(\frac{2}{3} \ln \left(\phi_{1 s}(t, \mathbf{p})\right)+\frac{1}{3} \ln \left(\phi_{1 h}(t, \mathbf{p})\right)-\frac{2}{3} \ln \left(\phi_{2 s}(t, \mathbf{p})\right)-\frac{1}{3} \ln \left(\phi_{2 h}(t, \mathbf{p})\right)\right)}{d t}=0$, where $\phi_{i \gamma}(t, \mathbf{p})$, corresponds to the probability that each agent $i$ assigns to strategy $\gamma$ at time $t$ given initial condition $\mathbf{p}$. This is a special case of corollary 4.9. We use this invariant function to identify the stable and unstable manifold of the interior Nash q.

Given any point $\mathbf{p}$ of the stable manifold of $\mathbf{q}$, we have that by definition $\lim _{t \rightarrow \infty} \phi(t, \mathbf{p})=\mathbf{q}$. Similarly for the unstable manifold, we have that $\lim _{t \rightarrow-\infty} \phi(t, \mathbf{p})=$ q. The time-invariant property implies that for all such points (belonging to the stable or unstable manifold), $\frac{2}{3} \ln \left(p_{1 s}\right)+\frac{1}{3} \ln \left(1-p_{1 s}\right)-\frac{2}{3} \ln \left(p_{2 s}\right)-\frac{1}{3} \ln \left(1-p_{2 s}\right)=\frac{2}{3} \ln \left(q_{1 h}\right)+\frac{1}{3} \ln (1-$ $\left.q_{1 h}\right)-\frac{2}{3} \ln \left(q_{2 h}\right)-\frac{1}{3} \ln \left(1-q_{2 h}\right)=0$, since the fully mixed Nash equilibrium is symmetric. This condition is equivalent to $p_{1 s}^{2}\left(1-p_{1 s}\right)=p_{2 s}^{2}\left(1-p_{2 s}\right)$, where $0<p_{1 s}, p_{2 s}<1$. It is straightforward to verify that this algebraic equation is satisfied by the following two distinct solutions, the diagonal line $\left(p_{2 s}=p_{1 s}\right)$ and $p_{2 s}=\frac{1}{2}\left(1-p_{1 s}+\sqrt{1+2 p_{1 s}-3 p_{1 s}^{2}}\right)$. Below, we show that these manifolds correspond indeed to the state and unstable man- 
ifold of the mixed Nash, by showing that this Nash equilibrium satisfies these equations and by establishing that the vector field is tangent everywhere along them.

The case of the diagonal is trivial and follows from the symmetric nature of the game. We verify the claims about $p_{2 s}=\frac{1}{2}\left(1-p_{1 s}+\sqrt{1+2 p_{1 s}-3 p_{1 s}^{2}}\right)$. Indeed, the mixed equilibrium point in which $p_{1 s}=p_{2 s}=2 / 3$ satisfies the above equation. We establish that the vector filed is tangent to this manifold by showing in Lemma 8.1 of the full version that $\frac{\partial p_{2 s}}{\partial p_{1 s}}=\frac{\frac{d p_{2 s}}{d t}}{\frac{d p_{1 s}}{d t}}:=\frac{p_{2 s}\left(u_{2}(s)-\left(p_{2 s} u_{2}(s)+\left(1-p_{2 s}\right) u_{2}(h)\right)\right)}{p_{1 s}\left(u_{1}(s)-\left(p_{1 s} u_{1}(s)+\left(1-p_{1 s}\right) u_{1}(h)\right)\right)}$, where the last equality is derived by the definition of replicator dynamics. Finally, this manifold is indeed attracting to the equilibrium. Since the function $p_{2 s}=y\left(p_{1 s}\right)=\frac{1}{2}\left(1-p_{1 s}+\right.$ $\left.\sqrt{1+2 p_{1 s}-3 p_{1 s}^{2}}\right)$ is a strictly decreasing function of $p_{1 s}$ in $[0,1]$ and satisfies $y(2 / 3)=$ $2 / 3$, this implies that its graph is contained in the subspace $\left(0<p_{1 s}<2 / 3 \cap 2 / 3<p_{2 s}<\right.$ 1) $\cup\left(2 / 3<p_{1 s}<1 \cap 0<p_{2 s}<2 / 3\right)$. In each of these subsets $\left(0<p_{1 s}<2 / 3 \cap 2 / 3<\right.$ $\left.p_{2 s}<1\right),\left(2 / 3<p_{1 s}<1 \cap 0<p_{2 s}<2 / 3\right)$ the replicator vector field coordinates have fixed signs that "push" $p_{1 s}, p_{2 s}$ towards their respective mixed equilibrium values.

The stable manifold partitions the set $0<p_{1 s}, p_{2 s}<1$ into two subsets, each of which is flow invariant since the unstable manifold itself is flow invariant. Our convergence analysis for the generalized replicator flow implies that in each subset all but a measure zero of initial conditions must converge to its respective pure equilibrium. The size of the lower region of attraction ${ }^{13}$ is equal to the following definite integral $\int_{0}^{1} \frac{1}{2}\left(1-p_{1 s}+\sqrt{1+2 p_{1 s}-3 p_{1 s}^{2}}\right) d x=\left[1 / 2\left(p_{1 s}-\frac{p_{1 s}^{2}}{2}+\left(-\frac{1}{6}+\frac{p_{1 s}}{2}\right) \sqrt{1+2 p_{1 s}-3 p_{1 s}^{2}}-\right.\right.$ $\left.\left.\frac{2 \arcsin \left[\frac{1}{2}\left(1-3 p_{1 s}\right)\right]}{3 \sqrt{3}}\right)\right]_{0}^{1}=\frac{1}{27}(9+2 \sqrt{3} \pi)=0.7364$ and the theorem follows.

\subsection{Average Price of Anarchy Analysis in Coordination/Consensus Games via Polytope Approximations of Regions of Attraction}

THEOREM 5.2. The average price of anarchy of a w-coordination game with $w \geq 1$ is at most $\frac{w^{2}+w}{w^{2}+1}$ and at least $\frac{w(w+1)^{2}}{w(w+1)^{2}-2 w+2}$.

Sketch. For $w$-coordination games it is straightforward to see that $p_{1 s}^{w}\left(1-p_{1 s}\right)-p_{2 s}^{w}(1-$ $p_{2 s}$ ) is an invariant property of the replicator system (follows from lemma 4.8). The presence of the parameter $w$ on the exponent precludes the existence of a simple, explicit, parametric description of all the solutions. We analyze the topology of the basins of attractions and produce simple subsets/supersets polytope approximations of them (see figure 2). The volume of these polytope approximations can be computed explicitly and these measures can be used to provide upper and lower bounds on the average case system performance and average price of anarchy. We present the complete proof in the full version of this paper.

By combining the exact analysis of the standard Stag Hunt game (theorem 5.1), theorem 5.2 and optimizing over $w$ we derive that:

COROLlaRY 5.3. The average price of anarchy of the class of $w$-coordination games with $w>0$ is at least $\frac{2}{1+\frac{9+2 \sqrt{3} \pi}{27}} \approx 1.15$ and at most $\frac{4+3 \sqrt{2}}{4+2 \sqrt{2}} \approx 1.21$. In comparison, the price of anarchy for this class of games is unbounded.

\footnotetext{
${ }^{13}$ This corresponds to the risk dominant equilibrium (Hare, Hare).
} 


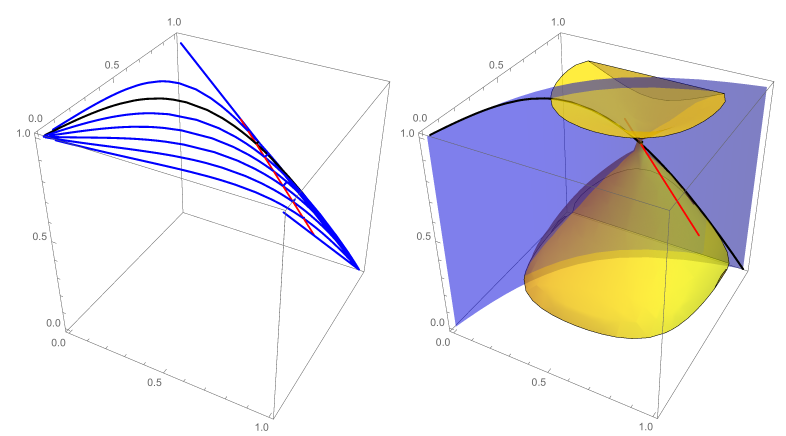

(a) Examples of stable man- (b) Stable manifolds lie on ifolds for different mixed the intersection of level sets Nash. of invariant functions.

Fig. 3. Star network coordination game with 3 agents

\subsection{Coordination/Consensus Games on a Star Graph}

In this subsection we show how to estimate the topology of regions of attraction for star networks of $w$-coordination games. This corresponds to strategic settings where some agents again need to reach consensus but where there is an agent who works as a center communicating with all agents at once. The price of anarchy and stability of these games remain unchanged as we increase the size of the star. Specifically the price of stability is equal to 1 whereas the price of anarchy can become arbitrarily large for large enough $w$. We will argue once again that the average performance is approximately optimal.

This game has two pure Nash equilibria where all agents either play the first strategy (i.e., Stag), or the second (i.e., Hare). For simplicity in notation sometimes we denote the first strategy, i.e., Stag, as strategy $A$ and the other strategy, i.e., Hare, as strategy $B$. This game has a continuum of mixed Nash equilibria. Our goal is to produce an oracle which given as input an initial condition outputs the resulting equilibrium that system converges to.

Example. In order to gain some intuition on the construction of these oracles let's focus on the minimal case with a continuum of equilibria ( $N=3$ agents/vertices, center agent with $n=2$ neighbors). Since each agent has two strategies it suffices to depict for each one the probability with which they choose strategy $A$ (the "bad" Stag strategy). Hence, the phase space can be depicted in 3 dimensions. Figure 3 depicts this phase space. The point $(0,0,0)$ captures the good pure Nash (all $B$ ), whereas the point $(1,1,1)$ the bad pure Nash (all $A$ ). There is also a continuum of unstable mixed Nash equilibria. Specifically, it suffices that the center player chooses $A$ with probability $w /(w+1)$ and the summation of the probabilities that the two other agents assign to $A$ is exactly $2 w /(w+1)$. In figure 3, we have chosen $w=2$. The continuum of equilibria corresponds to the red straight line. These are unstable equilibria and by Theorem 4.5 almost all initial conditions are attracted to the two attracting pure Nash. For any mixed Nash equilibrium there exists a curve (co-dimension 2) of points that converge to it. Figure 3(a) depicts several such stable manifolds for sample mixed equilibria along the equilibrium line. The union of these stable manifolds partitions the state space into two regions, one attracting to equilibrium $(A, A, A)$ and the other attracting to the equilibrium $(B, B, B)$ ). Hence, in order to construct our oracle it suffices to have a description of these attracting curves for the mixed equilibria. However, as shown in figure 3(b), we have identified two distinct invariant functions for the replicator dynamic in this system. Given any mixed Nash equilibrium, the set of points of the state space which 
agree with the value of each of these invariant functions define a set of co-dimension one (the double hollow cone and the curved plane). Any points that converge to this equilibrium must lie on the intersection of these sets (black curve). In fact, due to our point-wise convergence theorem, it immediately follows that this intersection is exactly the stable manifold of the unstable equilibrium. The case for general $N(=n+1)$ works analogously, but now we need to identify $N-1$ ( $=n$, equal to the number of neighbors) invariant functions in an algorithmic, efficient manner.

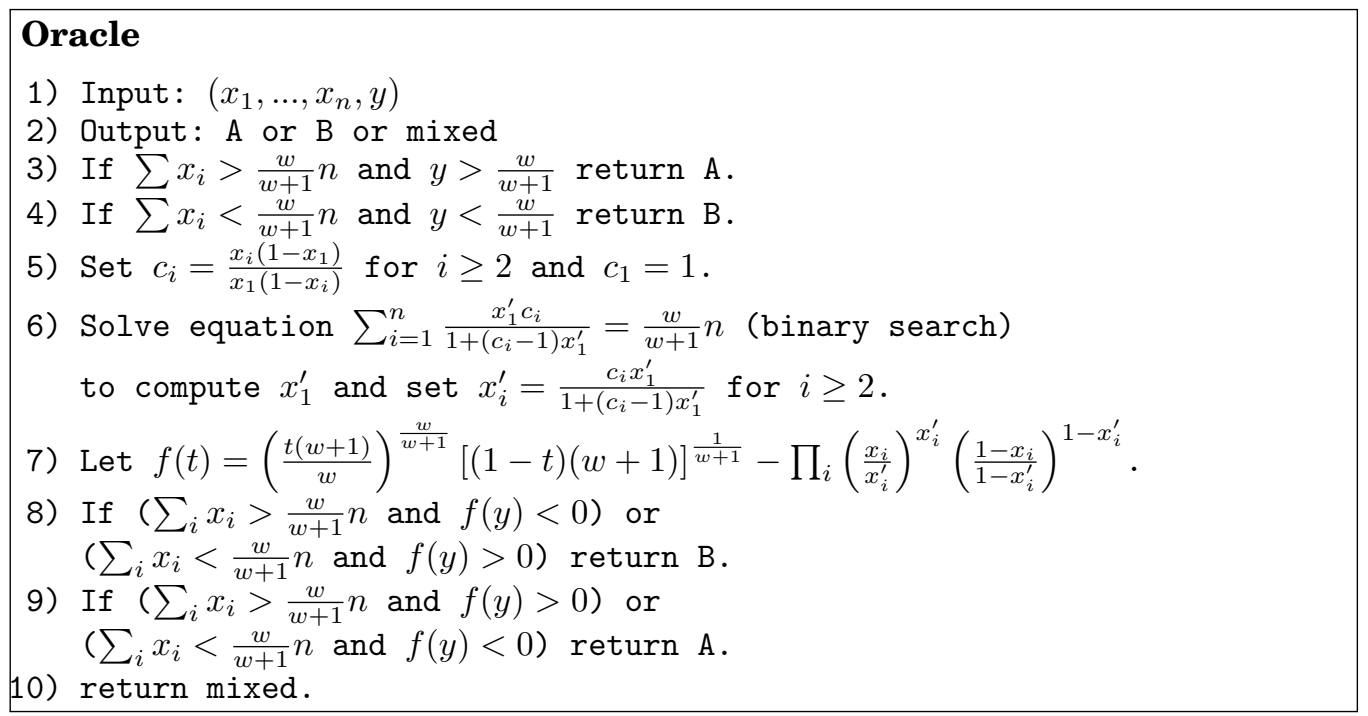

The proof of correctness of the algorithm is presented in the full version. Given this oracle it is straightforward to establish an upper bound of 1.868 for the average price of anarchy, which is independent both of $w$ as well as the size of the star.

COROLLARY 5.4. The average price of anarchy for the class of star w-coordination games (with $n+1$ agents) is at most 1.868 .

\subsection{Average price of anarchy of linear, symmetric load balancing games}

In this subsection, we state the following bounds on the average price of anarchy of linear, symmetric load balancing games.

THEOREM 5.5. The average price of anarchy in terms of makespan of symmetric, linear load balancing games is at most 3/2. Moreover, generically, the average price of anarchy of symmetric, linear load balancing games is 1 . Specifically, given any number of agents and machines, the set of linear latency functions such that the average price of anarchy of the resulting game is greater than 1 is a zero measure set within the set of all linear latency functions.

For the classic game of $n$-balls $n$-bins we can show the following theorem:

THEOREM 5.6. The average price of anarchy in terms of makespan for the (identical) n-balls n-bins is 1 .

\section{CONCLUSION AND OPEN QUESTIONS}

We define an average case analysis notion in dynamical systems focusing on games and replicator dynamics. We call this notion average price of anarchy (APoA) and provide upper and lower bounds for APoA in different classes of games. Several questions arise: 
- Other settings/games/mechanisms. In recent followup work, [Shnayder et al. 2016] applies our approach to peer prediction mechanisms where the size of the basin of attraction of the truthful equilibrium is used as a proxy for the robustness of truthful play. The replicator model predicts/confirms the significant improvement in robustness of recent mechanisms over earlier approaches. It would be interesting to test the robustness of other (approximately) truthful, differentially private mechanisms in a similar manner.

- Other dynamics. Perform average case analysis for other dynamics and compare them against replicator dynamics.

- Generalization of APoA. Generalize the notion of APoA to dynamics that do not necessarily converge. In particular, it would be intriguing to define an APoA notion for chain recurrent sets (see [Papadimitriou and Piliouras 2016]).

- Point-wise convergence. Generalize the point-wise convergence result to a larger class of congestion games, (e.g., for polynomial cost functions), as well as extend the point-wise convergence result for linear cost functions to other dynamics.

- Volumes of regions of attraction as a function. Given a prior distribution over initial conditions (e.g., uniform), every point-wise convergent dynamical system induces a probability distribution over fixed points. By approximating this function (from priors over initial conditions to posteriors over equilibria), we can predict the average case (long-term) behavior of the system (without having the equations of the dynamics). This interpretation of a game, as an experiment/measurement that maps the outside observer's original beliefs over initial mixed strategies/beliefs of the agents to (sampling from) a distribution/belief over the resulting equilibria, resolves the non-determinism problem linked to the multiplicity equilibria in a game. The unique, well-defined, deterministic prediction is the function from distributions over initial conditions to distributions over equilibria. Naturally, if the initial distribution is concentrated on a single equilibrium then the output of the prediction function will similarly be concentrated on that equilibrium. Nontrivial distributions will result to a (unique) distribution/prediction that puts positive measure on several equilibria. Developing a formal theory for approximating such functions seems like a fertile ground for combining ideas from computer science and game theory.

\section{REFERENCES}

H. Ackermann, P. Berenbrink, S. Fischer, and M. Hoefer. 2009. Concurrent Imitation Dynamics in Congestion Games. In PODC. 63-72.

A. Asadpour and A. Saberi. 2009. On the Inefficiency Ratio of Stable Equilibria in Congestion Games. In WINE. 545-552.

P. Berenbrink, T. Friedetzky, L. A. Goldberg, P. W. Goldberg, Z. Hu, and R. Martin. 2007. Distributed Selfish Load Balancing. SIAM J. Comput. (2007), 1163-1181.

P. Berenbrink, T. Friedetzky, I. Hajirasouliha, and Z. Hu. 2012. Convergence to Equilibria in Distributed, Selfish Reallocation Processes with Weighted Tasks. Algorithmica 62, 3-4 (2012), 767-786.

Y. Cai and C. Daskalakis. 2011. On Minmax Theorems for Multiplayer Games. In SODA. 217-234.

N. Cesa-Bianchi and G. Lugoisi. 2006. Prediction, Learning, and Games. Cambridge University Press.

C. Chung, K. Ligett, K. Pruhs, and A. Roth. 2008. The Price of Stochastic Anarchy. In SAGT. 303-314.

C. Daskalakis and C. H. Papadimitriou. 2009. On a Network Generalization of the Minmax Theorem. In ICALP. 423-434.

E. Even-Dar and Y. Mansour. 2005. Fast Convergence of Selfish Rerouting. In SODA. 
D. Fotakis, A. C. Kaporis, and P. G. Spirakis. 2008. Atomic Congestion Games: Fast, Myopic and Concurrent. In Algorithmic Game Theory. Lecture Notes in Computer Science, Vol. 4997. 121-132.

D. Fudenberg and D. K. Levine. 1998. The Theory of Learning in Games. The MIT Press.

J. C. Harsanyi and R. Selten. 1988. A General Theory of Equilibrium Selection in Games. Cambridge: MIT Press.

J. Hofbauer and K. Sigmund. 1998. Evolutionary Games and Population Dynamics. Cambridge University Press, Cambridge.

H. Khalil. 1996. Nonlinear Systems. Prentice Hall.

R. Kleinberg, K. Ligett, G. Piliouras, and É. Tardos. 2011. Beyond the Nash equilibrium barrier. In ICS.

R. Kleinberg, G. Piliouras, and É. Tardos. 2009. Multiplicative Updates Outperform Generic No-Regret Learning in Congestion Games. In STOC.

J. D. Lee, M. Simchowitz, M. I. Jordan, and B. Recht. 2016. Gradient Descent only Converges to Minimizers. In COLT, Vol. abs/1602.04915.

K. Ligett and G. Piliouras. 2011. Beating the best Nash without regret. SIGecom Exchanges 10 (2011).

V. Losert and E. Akin. 1983. Dynamics of Games and Genes: Discrete Versus Continuous Time. Journal of Mathematical Biology (1983).

R. Mehta, I. Panageas, and G. Piliouras. 2015. Natural Selection as an Inhibitor of Genetic Diversity: Multiplicative Weights Updates Algorithm and a Conjecture of Haploid Genetics. In ITCS.

R. Mehta, I. Panageas, G. Piliouras, P. Tetali, and V. V. Vazirani. 2015. Mutation, Sexual Reproduction and Survival in Dynamic Environments. ArXiv e-prints (2015). http://arxiv.org/abs/1511.01409

R. Mehta, I. Panageas, G. Piliouras, and S. Yazdanbod. 2014. The Complexity of Genetic . ArXiv e-prints (2014). http://arxiv.org/abs/1411.6322

D. Monderer and L. S. Shapley. 1996. Potential Games. Games and Economic Behavior (1996), 124-143.

R. B. Myerson. 1991. Game Theory: Analysis of Conflict. Harvard University Press.

N. Nisan, T. Roughgarden, E. Tardos, and V. V. Vazirani. 2007. Algorithmic Game Theory. Cambridge University Press.

I. Panageas and G. Piliouras. 2016. Gradient Descent Converges to Minimizers: The Case of Non-Isolated Critical Points. CoRR (2016). http://arxiv.org/abs/1605.00405

C. H. Papadimitriou and G. Piliouras. 2016. From Nash Equilibria to Chain Recurrent Sets: Solution Concepts and Topology. In ITCS. 227-235.

L. Perko. 1991. Differential Equations and Dynamical Systems. Springer.

G. Piliouras, C. Nieto-Granda, H. I. Christensen, and J. S. Shamma. 2014. Persistent Patterns: Multi-agent Learning Beyond Equilibrium and Utility. In AAMAS.

G. Piliouras and J. S. Shamma. 2014. Optimization Despite Chaos: Convex Relaxations to Complex Limit Sets via Poincaré Recurrence. In SODA.

T. Roughgarden. 2009. Intrinsic robustness of the price of anarchy. In STOC. 513-522.

W. H. Sandholm. 2010. Population Games and Evolutionary Dynamics. MIT Press.

V. Shnayder, R. Frongillo, and D. C. Parkes. 2016. Measuring Performance Of Peer Prediction Mechanisms Using Replicator Dynamics. In IJCAI.

H.P. Young. 2004. Strategic Learning and its Limits. Oxford University Press.

B. Zhang and J. Hofbauer. 2014. Equilibrium selection via replicator dynamics in 2x2 coordination games. International Journal of Game Theory 44, 2 (2014), 433-448. 